\title{
Co-trimoxazole and Prevention of Relapses of PR3-ANCA Positive VAsCulitis with Pulmonary InVOlVEMENT
}

\author{
K. Zycinska, K. A. Wardyn, T. M. Zielonka, R. Krupa, W. Lukas
}

\author{
Primary Systemic Vasculitis Outpatient Clinic, Department of Family Medicine, Internal and Metabolic Diseases, \\ Warsaw Medical University, Warsaw, Poland
}

\begin{abstract}
Background: Bacterial and viral respiratory tract infections may trigger relapses in patients with PR3-positive vasculitis. Data have suggested that treatment with co-trimoxazole may be beneficial, because this antibiotic could act by eliminating the offending microbe and thereby stopping the initiating stimulus.

Goal and methods: Prospective, randomized, placebocontrolled study of the efficacy of co-trimoxazole given $960 \mathrm{mg}$ thrice weekly for 18 months in preventing relapses in patients with Wegener's granulomatosis (WG) in remission, after treatment with cyclophosphamide and prednisolone was conducted. Relapses and infections were assessed with predefined criteria based on clinical, laboratory, serological, microbiological, and histopathological findings. Sixteen patients were assigned to receive co-trimoxazole and 15 to receive placebo.

Results: Seventy five percent of the patients in the cotrimoxazole group remained in remission at 18 months and $55 \%$ of those in the placebo group. A proportional hazard regression analysis identified a positive PR3-ANCA test at the start of treatment, chronic nasal crusting, and Staphylococus aureus infection as risk factors for relapse. Furthermore, the analysis identified treatment with co-trimoxazole as an independent factor associated with prolonged diseasefree interval.

Conclusion: Treatment with co-trimoxazole reduces the incidence of relapses in patients with Wegener's granulomatosis in remission.
\end{abstract}

Key words: co-tromoxazole, relapse, Wegener's granulomatosis

\section{INTRODUCTION}

Wegener's granulomatosis (WG) is an autoimmune disease characterized by necrotizing granulomatous inflammation of the upper and lower respiratory tract and necrotizing crescent glomerulonephritis. The pathogenesis of the disease is not well known. Patient with WG frequently have clinical symptoms of respiratory tract infection and the ANCA titer elevation [1]. Furthermore, they have frequent secondary infections of the nasal and paranasal tissue caused by Staphyloccocus aureus [2, 3].

A possible role for bacteria and viruses in recurrent Wegener's granulomatosis is suggested by studies of beneficial effects of co-trimoxazole [4, 5]. Trimetoprim and sulfamethoxazole (co-trimoxazole) inhibit bacterial synthesis of tetrahydrofolic acid, the physiologically active form of folic acid and co-factor in thymidine synthesis; thus bacterial DNA. Thrimetoprim-sulfametoxazol is available in oral and intravenous preparations. The standard single-strength tablet contains $80 \mathrm{mg}$ of trimetoprim and $400 \mathrm{mg}$ of sulfametoxazole, and the more clinically used doublestrength tablet contains $160 \mathrm{mg}$ of trimethoprim and $800 \mathrm{mg}$ of sulfametoxazole. When taken orally, both components are well absorbed from the gastrointestinal tract.

High concentration of both drugs is found in the sputum, cerebrospinal fluid, prostatic fluid, and bile [6]. Co-trimoxazol has been useful in the treatment of community-acquired upper and lower respiratory infections, because of its activity against the major pathogens Sreptococcus pneumoniae, Haemophilus influenzae, Moraxella caterrbalis, Staphylococcus aureus [7]. Moreover, it has proved beneficial for prophylaxis against opportunistic infections and for reduction in the occurrence of routine infections in patients receiving immunosuppressive therapy. Therefore, the aim of the present study was to assess efficacy of co-trimoxazole in preventing relapses in patients with Wegener's granulomatosis in remission, after prior treatment with cyclophosphamide and prednisolone.

\section{Material ANd Methods}

The study was approved by a local Ethics Committee. Prospective, randomized, placebo-controlled study of the efficacy of co-trimoxazole given $960 \mathrm{mg}$ thrice weekly for 18 months in preventing relapses in patients with WG in remission, after prior treatment with cyclophosphamide and prednisolone was conducted. Patients from Primary Systemic Vasculitis Outpatients Clinic of the Czerniakowski Hospital in Warsaw, Poland with biopsy-proven WG were entered the study. All patients fulfilled the American College of Rheumatology criteria for classification of WG and the Chapel Hill Consensus Conference definition, and also of the EUVAS ANCA-associated vasculitis definition for WG. Disease activity was confirmed by clinical scoring, laboratory variables, and imaging procedures. DEI and BVAS indexes were determined to measure organ involvement and disease activity. Relapses and infections were assessed with predefined criteria based on clinical, laboratory, serological, microbiological, and histopathological findings. 
Sixteen patients were assigned to receive co-trimoxazole and 15 to receive placebo. Patients were evaluated once every 3-4 months for signs of disease activity, infection, compliance with treatment regiment, and therapy side effects.

C-ANCA and PR3-ANCA were measured serially. Differences in frequencies of categorical variables between the two groups were studied by Fisher's test, and continuous variables were tested with a Wilcoxon test. Cox proportional hazard regression analysis was used to assessed other potential prognostic factors: age, gender, disease-free interval before the start of study, time since diagnosis, history of relapses, BVASWG and DEI indexes value, number of infections, type of infection, duration of infection, renal function measured as glomerular filtration rate (GFR), and nasal damage and structure. Hazard ratios (HR) are reported as relative risks with $95 \% \mathrm{Cl}$. A $\mathrm{P}$ value $<0.05$ was considered statistically significant.

\section{RESULTS}

Baseline characteristics of WG patients according to treatment are presented in Table 1 . In an analysis at 18 months of follow-up $75 \%$ of the patients in the cotrimoxazole group were in remission, as compared with $55 \%$ of the patients in the placebo group with HR 0.8 (95\% CI 0.21-1.20) (Table 2).

The proportional hazard regression analysis identified the use of co-trimoxazole $(\mathrm{P}=0.003)$ and the presence of PR3-ANCA at the start of study $(\mathrm{P}=0.004)$ as factors related to the disease-free interval with the respective relative risks of relapse of WG 0.4 (95\% CI, $0.12-0.69)$ and 2.56 (95\% CI, 1.41-6.35). A chronic nasal carriage of $S$. aureus was associated with persistent or intermittent c-ANCA or p-ANCA positivity $(\mathrm{HR}=2.68 ; \mathrm{Cl} 1.25-4.58)$. Relative risks for the occurrence of relapse were $6.15(\mathrm{Cl} 2.15-33.20)$ for chronic nasal carriage of S. aureus MRSA, 4.56 (Cl 2.45-7.65) for chronic nasal carriage of $S$. aureus MSSA, $3.45(\mathrm{Cl}$ 1.56-16.7) for nasal chronic crustation, $1.67(\mathrm{Cl} 1.37$ 6.35) for a glomerular filtration rate (GFR) $\leq 60 \mathrm{ml} / \mathrm{min}$, $1.87(\mathrm{Cl} \mathrm{0.98-4.02)}$ for refractory upper respiratory tract infection, and 1.21 (Cl 0.87-2.04) for relapses in anamnesis. The number of infections per year, type of infection (bacterial or viral, typical, or atypical) did not have a significant contribution. Factors related to the disease free interval in patients treated with co-trimoxazole or placebo are presented in Table 3.
Table 1. Baseline characteristics.

\begin{tabular}{lcc}
\hline Baseline Characteristics & $\begin{array}{c}\text { Co-trimoxazole } \\
\text { Group } \\
(\mathrm{n}=16)\end{array}$ & $\begin{array}{c}\text { Placebo } \\
\text { Group } \\
(\mathrm{n}=15)\end{array}$ \\
\hline Median age (yr) & $46.1(21.1-56.5)$ & $51.4(28.4-76.0)$ \\
Sex (M/F) & $8 / 8$ & $7 / 8$ \\
Disease duration (mo) & $67(8-86)$ & $66(7-71)$ \\
BVAS-WG & $8(2-11)$ & $11(12-14)$ \\
DEI & $4(3-8)$ & $5(3-7)$ \\
GFR (ml/min) & $81(74-110)$ & $71(486-88)$ \\
Length of remission (mo) & 15 & 16 \\
Positive serum test for PR3 & 7 & 9 \\
at the start of treatment & & \\
\end{tabular}

Table 2. Patients in remision.

\begin{tabular}{lrrr}
\hline Months & 0 & 12 & 18 \\
\hline \multicolumn{4}{c}{ \#. of patients in remission } \\
Co-trimoxazole & 16 & 14 & 12 \\
Placebo & 15 & 11 & 8 \\
\hline
\end{tabular}

The annual number of infections per patient was significantly lower in the co-trimoxazole group during the study than in the placebo group (median 0.0 vs. 4.0, $\mathrm{P}<0.01)$. Eight opportunistic infections were identified during the study. Six cases of herpes zoster (three in each group) and 2 cases of cytomegalovirus infection (both in placebo group).

At six months, the patients in the co-trimoxazole group had a median increase in the serum creatinine concentration of $21 \%$ over the baseline values (range $11-32 \%, \mathrm{P}<0.001)$, which persisted thoughtout the treatment period, and then returned to the baseline levels after the study end.

\section{Discussion}

The potential role of infectious factors in the induction and presence of Wagener's granulomatosis activity was first suggested by Fridrich Wagener in 1936 in the original paper [8]. According to this paper, disease is triggered by an infection of the upper respiratory tract to become then an allergic reaction to this infec-

Table 3. Factors related to the disease free interval in Wegener's granulomatosis patients.

\begin{tabular}{llll}
\hline Variable & HR & $95 \%$ Cl & P \\
\hline Co-trimoxazole & 0.4 & $0.12-0.69$ & 0.003 \\
Presence of PR3-ANCA at the start of study & 2.56 & $1.41-6.35$ & 0.004 \\
Chronic nasal carriage of Stapylococcus aureus MRSA & 6.15 & $2.15-33.2$ & 0.002 \\
Chronic nasal carriage of Stapylococcus aureus MSSA & 4.56 & $2.45-7.65$ & 0.001 \\
Nasal chronic crusting & 3.45 & $1.56-16.7$ & 0.0001 \\
Upper respiratory tract infection & 3.72 & $1.65-8.38$ & 0.0015 \\
Relapses in anamnesis & 1.07 & $0.89-1.217$ & 0.01 \\
GFR $\leq 60 \mathrm{ml} /$ min & 1.67 & $1.37-1.6 .35$ & 0.02 \\
\end{tabular}


tion. Others authors also have suggested a role for infection in induction relapses of WG [9-12].

The first observations of beneficial effects of treatment of WG with trimethoprim-sulfamethoxazol were published by DeRemee et al in 1985 [13]. Co-trimoxazole is particularly effective against aerobic gram-negative bacilli. Bacterial infections, especially in the nose and paranasal sinuses, where necrotic tissue serves as a favorable culture medium, frequently complicate WG $[13,14]$. Trimetoprim-sulfametoxazole reduces the severity of the infection and leads to clinical improvement, acting by eliminating the offending microbe and stopping the initiating stimulus. Co-trimoxazole could also act as an immunosuppressant in its own right as a folic acid antagonist [15-18].The results of the present study suggested that treatment with co-trimoxazole reduces the number of relapses in patients with WG in remission as it did in a study by Stegeman et al [19]. In the present study, at 18 months of a follow-up, $75 \%$ of patients in the co-trimoxazole group were in remission as compared with $55 \%$ of patients in the placebo group, with the HR 0.8 (95\% CI 0.21-1.20). In the Stegeman et al study [19], 41 patients were assigned to received cotrimoxazole and 40 to received placebo. In 8 of the 41 patients in the co-trimoxazole group $(20 \%)$, the drug had to be stopped because of side effects. According to a life-table analysis, $82 \%$ of patients in the co-trimoxazole group remained in remission at 24 months as compared with $60 \%$ of those in the placebo group (relative risk of relapse $0.4 ; 95 \% \mathrm{CI}, 0.17-0.98$ ) [19].

In the present study, we found no relation between the number of infections and the occurrence of relapses in WG. We also reported an association between the nasal carriage of $S$. aureus and increased risk of relapse in WG: Thus, it is tempting to postulate that treatment reduces the frequency of relapses by eliminating or reducing $S$. aureus in upper airways. Some authors have postulated that co-trimoxazole exerts anti-inflammatory effects by interfering with the formation of specific oxygen-derived radicals by activated neutrophils to mediate tissue destruction in WG. On the other hand, co-trimoxazole, through its antagonism of folic acid metabolism, may have immunosuppressive properties.

A drug used for a long-term prophylactic treatment must be safe and well tolerated, which is a feature of co-trimoxazole that has a low incidence of minor adverse effects. In a study by Stegeman et al [19], 20\% of patients in the co-trimoxazole group stopped treatment prematurely because of side effects. These adverse effects were mild, with the exception of an episode of presumed intestinal nephritis in one patient. The serum creatinine concentration increased during co-trimoxazole therapy, but this effect was reversible. We conclude that treatment with co-trimoxazole is safe and reduces the frequency of relapses in patients with Wegener's granulomatosis in remission.

Conflicts of interest: No conflicts of interest were declared by the authors in regard to this work.

\section{REFERENCES}

[1] Kallenberg CG. Pathogenesis of PR3-ANCA associated vasculitis. J Autoimmun 2008; 30 (1-2) 29-36.
[2] Popa ER, Stegeman CA, Abdulahad WH, van der Meer B, Arends J, Manson WM, Bos NA, Kallenberg CG, Cohen Tervaert JW. Staphylococcal toxic-shock-syndrome-toxin1 as a risk factor for disease relapse in Wegener's granulomatosis. Rheumatology (Oxford) 2007; 46 (6): 1029-1033.

[3] Popa ER, Stegeman CA, Bos NA, Kallenberg CG, Tervaert JW. Staphylococcal superantigens and T cell expansions in Wegener's granulomatosis. Clin Exp Immunol 2003; 132 (3): 496-504.

[4] Guilpain P, Guillevin L, Mouthon L. New insights into pathogenesis of ANCA-associated vasculitides. Presse Med 2007; 36(5): 854-859.

[5] Pagnoux C, Teixeira L. Wegener's Granulomatosis. Presse Med 2007; 36 (5): 860-887.

[6] Rodrigez-Pia A, Stone JH. Vasculitis and systemic infections. Curr Opin Rheumatol 2006; 18: 39-47.

[7] Masters PA, O’Bryan TA, Zurlo J, Miller DQ, Josi N. Trimethoprim-sulphamethoxazol revisited. Arch Intern Med 2003: 163: 402-410.

[8] Spagnolo P, Richeldi L, du Bois RM. Environmental triggers and susceptibility factors in idiopathic granulomatous diseases. Semin Respir Crit Care Med 2008; 29(6): 610-619.

[9] Lapraik C, Watss R, Bacon P, Carruthers D, Chakravarty K, D'Cruz D, Guillevin L, Harper L, Luqmani R, Mooney J, Scott D. BSR and BHPR guidelines for the management of adults with ANCA associated vasculitis. Rheumatology 2007; 1-11.

[10] Charier C, Henegar C, Launay O, Pagnoux C, Berezne A, Bienvenu C, Cohen P, Mouthon L, Guillevin L. Risk factors for major infections in Wegener's granulomatosis: analysis of 113 patients. Ann Rheum Dis 2009; 68(5): 658-663.

[11] Seo P. Wegener's granulomatosis: managing more than inflammation. Curr Opion Rheumatol 2008; 20(1): 10-16.

[12] Lee JH, Attygalle T, Gaffney K, Scott DG. Demograph ics and environmental factors in Wegener's granulomatosis cluster. Ann Rheum Dis 2007; 66 (2): 278-279.

[13] DeRemee RA, McDonald TJ, Weiland LH. Wegener's granulomatosis: observations on treatment with antimicrobial agents. Mayo Clin Proc 1985; 60: 27-32.

[14] Gottschlich S, Ambrosch P, Kramkowski D, Laudien M, Buchelt T, Gross WL, Hellmich B. Head and neck manifestations of Wagener's granulomatosis. Rhinology 2006; 44(4): 227-233.

[15] DeRemee RA. Trimetoprim-sulphamethoxazole for the treatment of Wegener's granulomatosis. Rheumatology 2003; 42(2): 396-397.

[16] Rasmussen N, Petersen J, Remvig L, Andersen RV. Treatment of Wegener's granulomatosis with trimethoprimsulfamethoxazole. APMIS Suppl 1990; 19: 61-62.

[17] Seo P. Vasculitis: lessons learned. Curr Opin Rheumatol 2009; 21(1): 1-2.

[18] Jayne D. Progress of treatment in ANCA-associated vasculitis. Nephrology 2009; 14(1): 42-48.

[19] Stegeman C, Tervaert JWC, De Jong PE. Trimethoptimsulfamethoxazole (Co-trimoxazole) for the prevention of relapses of Wegener's granulomatosis. NEIM 1996: 335: $16-20$

\section{Author's address: \\ K. Zycinska}

Primary Systemic Vasculitis Outpatient Clinic

Department of Family Medicine, Internal and Metabolic Diseases, Nephrology Unit

Warsaw Medical University

Stepinska 19/25 St.,

00-187 Warsaw, Poland

Phone/Fax: +48223186325

E-mail: kzycinska@poczta.fm 